\title{
NEURAL CONTROL OF ECTOPIC FILIFORM SPINES IN ADULT TONGUE
}

\author{
B. OAKLeY, L.-H. Wu, A. LAWTON and C. DeSibour \\ Department of Biology, University of Michigan, Ann Arbor, MI 48109, U.S.A.
}

\begin{abstract}
The tongue surface directly above a fungiform taste bud is flat, thinly keratinized, and free of filiform spines. We examined fungiform papillae in serial sections of rat and gerbil tongues after unilateral transection of the chorda-lingual nerve had caused many fungiform taste buds to degenerate. Such empty fungiform papillae often formed a solitary keratinized outgrowth that closely resembled the spine of an ordinary filiform papilla. By six months an ectopic spine was found on $61 \%$ of empty fungiform papillae, but never on fungiform papillae that contained a taste bud. Experimental innervation of the tongue reduced the incidence of ectopic filiform spines in proportion to the cross-sectional area of the trigeminal nerve branches tested (the mylohyoid nerve, the lingual nerve, lingual + mylohyoid or lingual + auriculotemporal nerves). The chorda tympani nerve was 60 times more effective than trigeminal nerves in preventing ectopic filiform spines.

We suggest that positive and negative trophic actions are normal characteristics of taste axons, for they promote the formation of taste buds and prevent the expression of ectopic filiform spines. By preventing the outgrowth of ectopic spines on fungiform papillae, taste axons maintain a thinly keratinized apical surface that can be breached by the taste receptor cells.
\end{abstract}

While it has been suspected that many oral structures are morphologically dependent upon innervation, clear evidence for the neural control of the development and maintenance of oral structures exists only for taste buds. ${ }^{7.14}$ In this study, we provide evidence that innervation represses the outgrowth of filiform spines from fungiform papillae.

Thousands of keratinized conical spines normally cover the edge and dorsal surface of the tongue of many vertebrates. These are the filiform spines responsible for the rasp-like surface of mammalian tongues. The characteristic caudal curvature of filiform spines makes licking and lapping more effective. Filiform spines occur with densites exceeding $100 / \mathrm{mm}^{2}$ at the tip of the albino rat's tongue, with lesser densities caudally. Each filiform papilla consists of a keratinized spine rising from the apex of a dome-like protrusion or papilla of the lamina propria. This connective tissue dome is capped by several upwardly migrating columns of basal cell progenykeratinocytes that blend into the conical filiform spine. ${ }^{8}$ The packed external layers of the protruding spine are formed by these keratinocytes as they progressively flatten and slough off. ${ }^{3.8,9}$ Analogous proliferation columns are believed to be responsible for cell replacement in the hairy skin of mice, where the basal stem cells of each "epidermal proliferative unit" generate an upwardly migrating stack of flattened progeny that comprise the hexagonal keratinized squames of the skin surface. ${ }^{16}$

Abbreviations: PBS, phosphate-buffered saline; PBSTX, PBS containing $0.4 \%$ Triton X-100.
In development, rat filiform spines and their underlying papillae continue to enlarge even after innervation is eliminated. When the smooth epithelium of fetal tongue was explanted to the anterior chamber of the eye, the filiform papillae continued to develop and form spines. ${ }^{4.6}$ Similarly, filiform papillae developed normally if, before filiform spines emerged, anlage or fragments of the tongue were placed at embryonic days 12-14 (E12-14) into a nerve-free organ culture. ${ }^{1.5}$

Each fungiform papilla in rodents consists of a wide, apically protruding, connective tissue column that supports a solitary taste bud. The apical surface of the fungiform papilla is flat, thinly keratinized, and breached by the tip of its taste bud. When the rat or gerbil tongue is viewed from above, the nearly 90 fungiform papillae per side resemble scattered, minute circular clearings in a dense forest of caudally curving filiform spines. Although the beginnings of rat fungiform papillae are clearly evident at E15 before the invasion of axons at E16-17, ${ }^{11}$ adequate innervation seems to be required for further normal development. Fungiform papillae fail to mature after fragments of fetal rat tongue are explanted to the anterior chamber of the eye at E14-15.4

We examine the structure, incidence and neural control of the expression of ectopic filiform spines on empty fungiform papillae.

\section{EXPERIMENTAL PROCEDURES}

Surgical procedures: interruption of the chorda-lingual nerve

The chorda tympani and the lingual nerves unite to form the chorda-lingual nerve. Their removal eliminates the majority of sensory fibers to the anterior two-thirds of the rodent tongue; some autonomic fibers remain. In all 
operations to alter lingual innervation we anesthetized rodents with ketamine and $1 \mathrm{mg}$ of sodium pentobarbital i.p. The dosage of ketamine was $0.2 \mathrm{mg} / \mathrm{g}$ body weight for rats and $0.3 \mathrm{mg} / \mathrm{g}$ body weight i.m. for gerbils. By the second day of postoperative recovery. the animals were eating and drinking normally.

Rats (Sprague-Dawley albino from Harlan Co., Indianapolis, IN). The chorda tympani and lingual nerves were unilaterally transected, either where they unite near the auditory bulla ( $n=6$ rats) or several millimeters more distally where the combined chorda-lingual nerve approaches the tongue medial to the mandible $(n=40)$. In the latter procedure the proximal portion of the transected chorda-lingual nerve was sutured to the distal portion of the nearby mylohyoid nerve to encourage chorda-lingual axons to regenerate towards the normal targets of the mylohyoid nerve and discourage them from returning to the tongue.

A second operation, eight days before being killed. intended to interrupt regenerating chorda-lingual axons along its pathway near the mandible did not produce more extensive taste bud degeneration $(n=4$ of the 40 rats). To rule out the influence of IXth nerve axons, in 18 out of the 40 rats both IXth nerves were transected near the carotid bifurcation at the time the chordalingual nerve was unilaterally interrupted. The distal portion of the IXth nerve stumps was avulsed. Since these variations in nerve interruption produced equivalent and widespread fungiform taste bud degeneration, the groups have been combined in the presentation of the results.

Mongolian gerbils (Meriones unguiculatus from Tumblebrook Farms, West Bloomfield, MA) were used. In 12 gerbils the chorda-lingual nerve was transected unilaterally near the mandible. Three of these gerbils were killed eight days later, while in nine gerbils the chorda-lingual pathway was incised again at 14 days to eliminate putative regenerating axons. These nine gerbils were subsequently killed at 22 days.

Surgical procedures: trigeminal innervation of fungiform taste buds in rats

To determine whether somatosensory or motor axons would reduce the incidence of ectopic filiform spines we used three trigeminal (Vth cranial nerve) branches: the lingual nerve, which supplies somatosensory fibers to the anterior two-thirds of the tongue, the auriculotemporal nerve, which provides somatosensory axons to the pinna and side of the face; and the mylohyoid nerve, which supplies somatosensory axons to the skin of the chin and motor axons to three neck muscles. These three trigeminal nerves were surgically exposed near the union of the lingual and chorda tympani nerves. Four patterns of experimental innervation were made on the right side by partial denervation or by uniting severed nerve stumps with a single $11-0$ nylon suture (Ethicon Co.). (1) L (lingual nerve), $n=5$ rats. The chorda tympani nerve was transected and the proximal portion avulsed. leaving the lingual nerve intact. (2) $\mathrm{L}+\mathrm{MH}$ (lingual and mylohyoid nerves), $n=5$. The proximal stump of the transected mylohyoid nerve was sutured to the distal stump of the chorda lympani and the proximal chorda tympani was avulsed. The lingual nerve remained intact. (3) MH (mylohyoid nerve), $n=10$. The procedure for $\mathrm{L}+\mathrm{MH}$ was followed, except that the ipsilateral lingual nerve was also transected and deflected. Twenty-eight days after surgery the silver-stained tongues of five out of 10 rats were examined to determine the success of mylohyoid axons in innervating fungiform papillae. (4) $L+A T$ (lingual and auriculotemporal nerves), $n=4$. The auriculotemporal nerve was sutured to the distal stump of the chorda tympani nerve and the proximal stump of the chorda tympani was avulsed. The lingual nerve remained intact.

\section{Histological methods}

Animals were deeply anesthetized with an i.p. injection of sodium pentobarbital. An infusion pump was used to perfuse the anesthetized animals intracardially with $250 \mathrm{ml}$ of buffered $0.9 \% \mathrm{NaCl}(\mathrm{pH} 7.4)$ containing $0.02 \%$ heparin and $0.5 \%$ procaine $\mathrm{HCl}$, followed by a $250 \mathrm{ml}$ solution of either $4 \%$ formaldehyde, $1 \% \mathrm{NH}_{4} \mathrm{OH}$ and $15 \%$ sucrose, or for immunocytochemistry $4 \%$ paraformaldehyde in $0.1 \mathrm{M}$ phosphate-buffered saline (PBS).

Serial, $10-\mu \mathrm{m}$ transverse sections of paraffin embedded tongues of 59 rats and five gerbils were stained with Heidenhain's iron hematoxylin. To evaluate the entire fungiform field we examined serial sections of each tongue from the tip to the giant conical papillae near the dorsal median eminence. ${ }^{10}$

After unilateral interruption of the chorda-lingual nerve, lingual axons in serial, $40-\mu \mathrm{m}$ sagittal frozen sections were stained by Winkelmann's silver procedure ${ }^{16}$ which allowed us to trace axons into the papillae to the normal site of taste buds. Tongues were examined at eight days ( $n=3$ gerbils), 22 days ( $n=4$ gerbils), 28 days ( $n=4$ rats), and 180 days $(n=2$ rats), or 180 days after ipsilateral reinnervation of the operated side of the tongue with the mylohyoid nerve $(n=5$ rats, $\mathrm{MH}$ group).

Samples of the chorda tympani, lingual, mylohyoid and auriculotemporal nerves were obtained from three normal rats in the region beneath the mandible where the nerve crossing operations were carried out. Cross-sections of two to three samples of each type of nerve per animal were examined in a scanning electron microscope (Hitachi S-570). after the tissue had been dehydrated with hexamethyldisilazane and gold-coated. A graphics tablet and microcomputer were used to measure the cross-sectional areas of the scanning electron micrographs of the nerve samples, excluding the perineurium.

Immunocytochemistry. The proximal stump of the transected chorda-lingual nerve was sutured to the distal stump of the mylohyoid nerve and both IXth nerves were transected in 12 rats whose tongues were to be examined for keratin-like immunoreactivity. Rats were anesthetized with sodium pentobarbital and perfused intracardially, as described above, at 0 days $(n=1)$, eight days $(n=2), 21$ days $(n=2), 28$ days $(n=2), 35$ days $(n=2), 120$ days $(n=1)$ and 180 days $(n=2)$ after surgery. Tongues were excised, placed in $4 \%$ paraformaldehyde overnight, transferred into $20 \%$ sucrose in PBS and then into $30 \%$ sucrose in PBS until the tissue sank. Cryostat sections, $10 \mu \mathrm{m}$ thick, were placed on formaldehyde-treated gelatin-coated slides, air-dried and refrigerated at 4 C.

Slides with mounted tissue sections were washed in PBS containing $0.4 \%$ Triton $\mathrm{X}-100$ (PBSTX) followed by a 30-60 min exposure to $3 \%$ normal goat serum in PBSTX (Cappel, Cooper Biomedical) and a $1 \mathrm{~h}$ incubation with the primary rabbit antibody against bovine keratin (1:100 dilution in 3\% nomal goat serum in PBSTX: Axell AXL745, Accurate Chemical and Scientific Corp). The tissue sections were rinsed in PBS after this and each subsequent step. Following incubation for $45-60 \mathrm{~min}$ with biotin. labeled goat secondary antibody to rabbit $\operatorname{lgG}(1: 200$ in PBS; Sigma), the sections were exposed to Vectastain avidin-biotin complex for $30-60 \mathrm{~min}$ (Vector Laboratories)

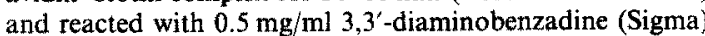
and $0.01 \%$ hydrogen peroxide and $0.01 \% \mathrm{NiCl}_{2}$ in PBS

\section{Identification of fungiform papillae by size}

In transverse sections at all rostrocaudal levels of the tongue we determined the mean area of representative fungiform and filiform papillae on both the normal and operated sides. We defined the area of a papillae in transverse section as the area of a dome of the lamina propria delimited by the row of basal cells along the papilia's apical and lateral margins, enclosed at the base of the papillae by a straight line. This definition excluded the area of taste buds 
and spines. A papilla's mean area was an average of microcomputer assisted areal measurements of camera lucida drawings of the three to four transverse sections passing near the papilla's center. The ratio of the mean area of a fungiform papilla to the mean area of the largest of the several nearby filiform papillae was calculated for pairs of fungiform and filiform papillae. As described in the results, the fungiform papillae, even those without a taste bud as a marker, were readily identified because they were an average of four to five times larger than neighboring filiform papillae. No papilla lacking a taste bud was tallied as a fungiform papilla unless its area was at least twice that of the largest neighboring filiform papilla.

\section{RESULTS}

Normal fungiform papillae always had a taste bud and never had an ectopic spine. Unilateral interruption of the chorda-lingual nerve caused many fungiform taste buds to degenerate. Those taste buds that were present after interruption of the chorda-lingual nerve were abnormal; they were either atrophic, averaging about half of their normal volume, or remnants, averaging about a quarter of their normal volume and lacking the ovoid taste bud shape and the elongated fusiform cells characteristic of both normal and atrophic taste buds. Following the complete disappearance of many fungiform taste buds, some of these empty fungiform papillae appeared to grow a solitary filiform-like spine from their apical surface (Fig. 1A-C).

The occurrence of ectopic filiform-like spines was not peculiar to rats. Gerbils had ectopic spines that were similar to those of rats, both in their curved, conical form and in their incidence; after interruption of the chorda-lingual nerve $14 \%$ of empty fungiform papillae in five rats had a prominent spine 21 days after surgery, compared with a spine on $18 \%$ of empty papillae in nine gerbils at 22 days (Fig. IF). In both gerbils and rats, ectopic spines never appeared in the presence of normal or atrophic taste buds (Fig. 1D), although this rule could not be as well established in gerbils because atrophic fungiform

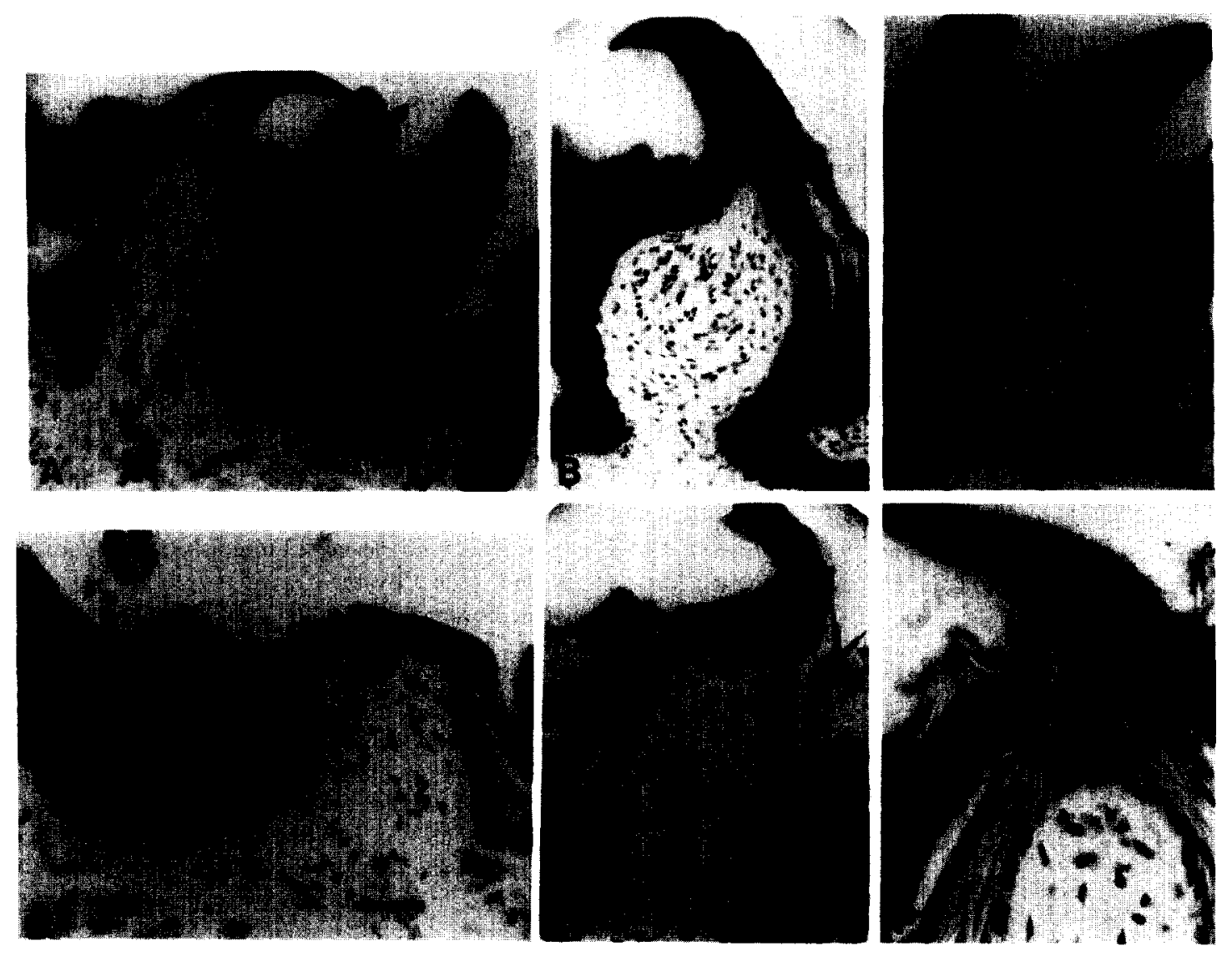

Fig. 1. (A-F) Transverse sections of rat tongue. (A) This large filiform-like, laterally pointing, spine was present on an empty fungiform papilla 180 days after transection of the chorda tympani nerve. The proximal portion of two normal, caudally directed, filiform spines are visible on the right. (B) A laterally directed, ectopic filiform-like spine is shown at 21 days and (C) at 28 days, after interruption of the chorda-lingual nerve in rats. (D) A fungiform papilla with an atrophic taste bud lacked a filiform-like outgrowth 180 days after interruption of the chorda-lingual nerve. (E) A fungiform papilla, containing a large remnant of a taste bud, had a distorted spine 28 days after interruption of the chorda-lingual nerve. (F) A spine was present on a gerbil fungiform papilla 22 days after interruption of the chorda-lingual nerve. Scale bar in $\mathrm{D}=33 \mu \mathrm{m}$ for $\mathrm{A}, \mathrm{D} ; 40 \mu \mathrm{m}$ for $\mathrm{B}, \mathrm{C}, \mathrm{E} ; 17 \mu \mathrm{m}$ for $\mathrm{F}$. 
taste buds were uncommon. probably owing to the more effective exclusion of trophic axons.

We examined tongues eight days after surgery to determine whether ectopic spines began to develop promptly after chorda-lingual nerve interruption or were the product of a slow epithelial transformation. Some developing ectopic spines were observed as early as eight days, scattered throughout the fungiform field (Fig. 2). At the next time point sampled (21 days) ectopic spines were more abundant and were now detectable at the tip of the tongue, where filiform spines are never large, and ectopic spines were too small to be readily detected at eight days.

\section{Evidence that spines emerged from fungiform papillae}

In this section we consider in detail the evidence that empty fungiform papillae developed an ectopic spine after taste bud degeneration. Among the most telling evidence that ectopic spines emerged from fungiform papillae were instances during the first postoperative month when a filiform-like spine and a remnant of a taste bud occurred in the same papilla (Fig. IE). These unusual associations of a spine and taste bud remnant must have been unstable, for spines were never associated with any form of taste bud at six months after surgery. The hypothesis that empty fungiform papillae formed new spine-like outgrowths is consistent with their increasing prevalence over time. By 180 days after surgery the incidence of identified empty fungiform papillae with a definite ectopic spine reached $61 \%$ (Fig. 3), and the distribution of fungiform papillae with ectopic spines resembled the distribution of normal fungiform papillae.

A separate avenue of evidence that fungiform papillae developed ectopic spines is the measurement of the relative size of fungiform and filiform papillae. Normal fungiform papillae were readily identifiable hy their large size; they were wider and had a much greater apical protrusion than the narrow and less extended filiform papillae. The lightly stained, cell-

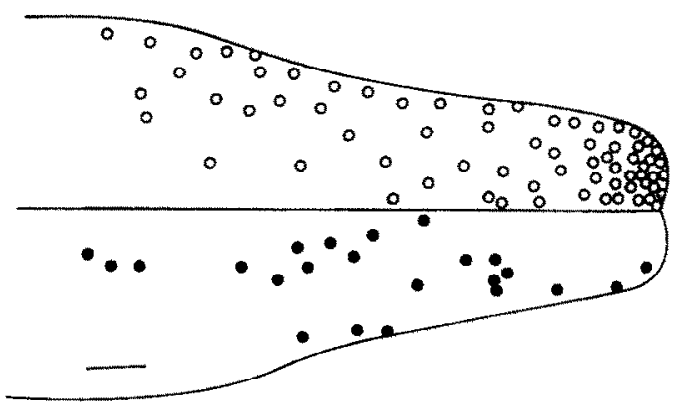

Fig. 2. The distribution of fungiform papillae (open circles) on the left half of the anterior two-thirds of a normal gerbil's tongue was reconstructed from serial $10 \mu \mathrm{m}$ transverse sections. The distribution of empty fungiform papillae with a filiform-like spine (filled circles) was reconstructed from $40 \mu \mathrm{m}$ silver-stained sagittal sections in this composite from three gerbils eight days after interruption of the chordalingual nerve. Scale bar $=1 \mathrm{~mm}$.

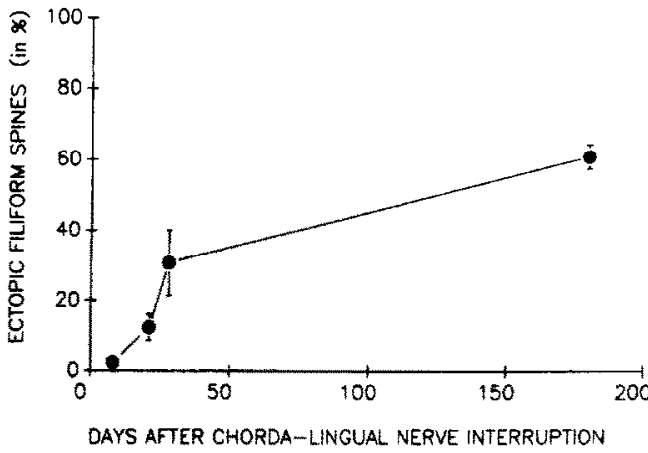

Fig. 3. The percentage of empty fungiform papillae with an ectopic filiform spine increased with days since interruption of the chorda-lingual nerve. The data points are the mean \pm S.E. percentage in five to seven rats of fungiform papillae with an ectopic spine. The data point at 180 days represents 90 spine-bearing fungiform papillae in a total of 148 empty fungiform papillae. The mean number of empty fungiform papiliae varied by $15 \%$ over the eight to 180 -day period.

sparse, connective tissue cores of both kinds of papillae are apparent in Figs $1 \mathrm{~A}$ and $4 \mathrm{C}$. The mean transverse area of normal fungiform papillae was $5.0 \pm 1.4$ times greater than that of nearby filiform papillae (mean \pm S.D., $n=101$ normal fungiform/filiform pairs, $P<0.001, t$-test between mean areas of fungiform and filiform papillae of six rats, see Experimental Procedures). There was less than $10 \%$ variation in the mean fungiform to filiform area ratios among four different rostrocaudal levels of the fungiform field $(20+$ pairs/level). Six months after interruption of the chorda-lingual nerve, the mean area of fungiform papillae with an atrophic taste bud was $4.8 \pm 1.4$ times larger than the area of nearby filiform papillae ( 50 fungiform/filiform pairs in six rats, $P<0.001, t$-test). Even when interruption of the chorda-lingual nerve eliminated the taste bud as a useful marker, empty fungiform papillae that lacked a spine could be clearly distinguished from nearby filiform papillae by their larger size and flat apical surface. For an ectopic spine to be tallied we required that the fungiform papilla with the ectopic spine be at least twice as large as the largest neighboring filiform papilla. Two observations demonstrated the conservatism of this stipulation. Firstly, the mean area of empty fungiform papillae with an ectopic spine was $4.0 \pm 1.2$ times larger than the area of neighboring filiform papillae (for 64 pairs of papillae in six rats at six months, $P<0.001, t$-test) (Fig. $4 C$ ). Secondly, a filiform papilla twice the area of other nearby filiform papillae would have been more than three standard deviations above the mean filiform papilla area. The probability of a filiform papilla that large was less than 0.001 , given a normal sampling distribution of filiform papilla areas on the operated side with a mean \pm S.D. of $32,800 \pm 9600 \mu \mathrm{m}^{2}$, $n=50$ filiform papillae in six rats at six months. Hence, a two-fold size difference for tallying spinebearing fungiform papillae was a conservative crite- 

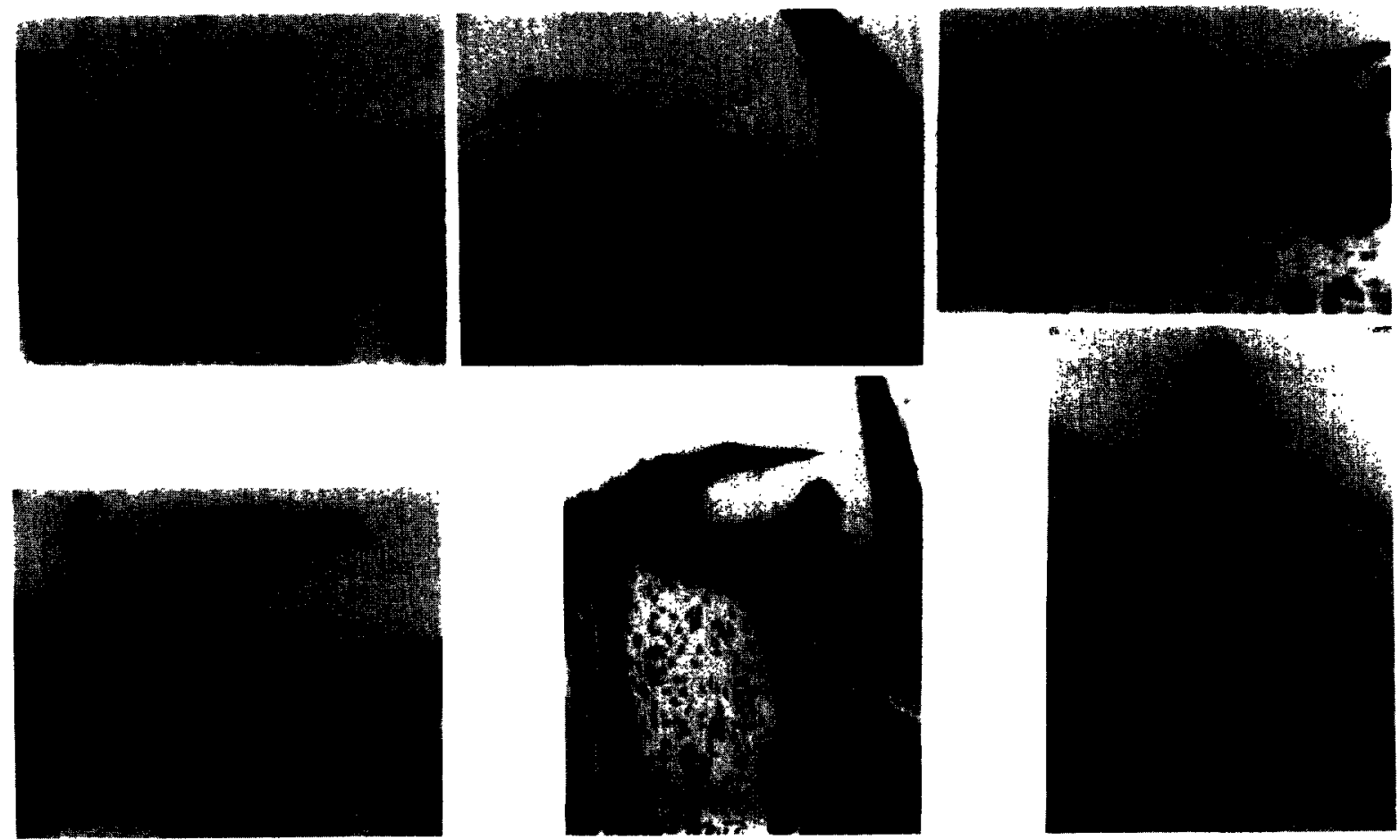

Fig. 4. Staining with a polyclonal antibody to keratin revealcd a stained core and an unreactive exterior of compacted layers of keratinocytes both in A a normal, caudally-curving, filiform spine and in D a laterally directed ectopic filiform spine, each 35 days after interruption of the chorda-lingual nerve. (B) Silver-stained axons (arrows) are present in a fungiform papilla with a small ectopic spine; Methyl Green counterstaining. (C) Near the tip of the tongue is a small fungiform papilla with a blunt, eccentric, ectopic spine and two flanking filiform spines-all pointing caudally. (E) A sharply curved, small ectopic filiform spine points medially toward a caudally curving, normal filiform spine. (F) A fungiform papilla is amply innervated by axons of the mylohyoid nerve - the chorda-lingual nerve had been interrupted 28 days earlier. Scale bar in $\mathrm{E}=40 \mu \mathrm{m}$ for $\mathrm{A}-\mathrm{F}$. $\mathrm{A}-\mathrm{C}$ and $\mathrm{F}$ are sagittal sections. $\mathrm{B}, \mathrm{C}, \mathrm{E}$ are 80 days after interruption of the chorda-lingual nerve.

rion unlikely to result in the tally of a normal filiform papilla by mistake.

Apart from the documented larger size of fungiform papillae, there were several qualitative characteristics that independently served to identify empty fungiform papillae with ectopic filiform-like spines. Several spine-bearing fungiform papillae were independently identifiable by the atypical orientation or shape of their ectopic spine. Rather than curving in the normal caudal direction, nearly half of the ectopic filiform spines curved laterally or medially, making them obvious in transverse sections of the tongue (Figs 1A-C, 4D,E). In addition, unlike ordinary filiform spines, ectopic spines were occasionally spindly and sharply curved (Fig. 4B,E), blunt or eccentrically located on the papilla (Fig. 4C). Such differences in spine orientation and shape provided independent verification that these were not ordinary spines on filiform papillae, but were ectopic spines on fungiform papillae.

The four-fold larger size of empty fungiform papillae with an ectopic spine coupled with instances of atypical spine orientation or shape, led to reliable identification of all but some of the smallest empty fungiform papillae with an ectopic filiform-like spine.
When, on occasion, the fungiform papilla was sharply tilted, analysis of complete serial sections eliminated the possibility of mistaking the spine of an adjacent filiform papilla for an ectopic spine. Generally, ectopic spines emerged from the center of a fungiform papilla, which allowed a full view of both the fungiform papilla and spine in the same histological section. We concluded that interruption of the chorda-lingual nerve caused many empty fungiform papillae to develop a pronounced ectopic filiform-like outgrowth on their apical surface.

\section{Evidence that the outgrowths from fungiform papillae were a form of filiform spine}

Both the typical cctopic filiform-like spine, (e.g. Fig. 1A-C) and normal filiform spines (Figs 1A,C, 4B,C,E) consisted of a protruding, pointed, conical peg covered by compact layers of flattened keratinocytes. Frequently, the dissociation of the packed keratinocytes revealed multiple, external keratin layers characteristic of normal filiform spines (Figs IC, F, 4A,B,F). For both normal and ectopic spines, keratin-like immunoreactivity was intense in the core of the spine with less staining within the compacted, superficial layers (Fig. 4A,D). In spite of the 
occasional instances of mishapen spines noted above. the structure, immunoreactivity and overall similarity in shape, make it appropriate to consider these new outgrowths as ectopic filiform spines.

Gustatory and trigeminal nerve control of the expression of ectopic filform spines

Gustatory innervation and/or the presence of taste buds prevented the outgrowth of an ectopic spine, for spines never co-existed in the same fungiform papilla with normal or atrophic taste buds (Fig. ID). Ectopic filiform spines were also absent when a remnant of a taste bud was present, except for their occasional, revealing co-occurrence within one month after surgery (Fig. IE).

An impression that ectopic spines were smaller or occurred less often after axons entered an empty fungiform papilla prompted an experimental test to determine whether axons from various trigeminal branches would reduce the incidence of ectopic filiform spines. We innervated the tongue with four combinations of trigeminal nerve branches: mylohyoid, lingual, lingual + mylohyoid and lingual + auriculotemporal nerves. In the last three groups, axons of the lingual nerve were known to be present, as the lingual nerve had never been manipulated. Silver staining of axons verified that mylohyoid axons successfully invaded the fungiform papillae. After interruption of the chorda-lingual nerve, innervation of the tongue with only the mylohyoid nerve greatly augmented the number of axons in most fungiform papillae observed at 28 days (Fig. 4 F). Such trigeminal innervation reduced the percentage of empty fungiform papillae with an ectopic fliform spine from a maximum of $61 \%$ with the fewest axons present $(-\mathrm{CL})$, to $30 \%$ with the attempt to maximize trigeminal nerve innervation (L+AT; Fig. 5). The lingual nerve was no more effective in spine suppression than the mylohyoid or auriculotemporal nerves. Note the agreement between the percentage of empty fungiform papillae that developed an ectopic spine after interruption of the chorda-lingual nerve $(61 \%)$ and the percentage expected with zero trigeminal innervation as given by the $y$-intercept $(58 \%)$. The $x$-intercept predicts that trigeminal nerve branches would require an aggregate cross-sectional area of $126,000 \mu \mathrm{m}^{2}$ to prevent all ectopic filiform spines. This is 60 times the area of the chorda tympani nerve which by itself will prevent expression of any ectopic filiform spines, while supporting all fungiform taste buds. ${ }^{13}$

\section{DISCUSSION}

Several lines of evidence indicated that interruption of the chorda-lingual nerve caused fungiform papillae to develop an ectopic filiform-like spine. One important observation was the formation of an ectopic spine on the apical surface of fungiform papillae independently identified by the presence of a taste bud

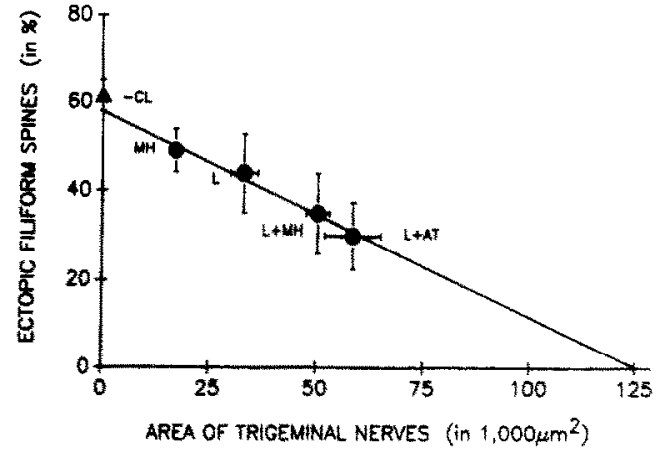

Fig. 5. The percentage of empty fungiform papillae with a filiform spine (mean $\pm S . E$.) decreased as a linear function of the cross-sectional area of the Vth nerve branches used to innervate the rat tongue. The $x$-coordinate of each filled circle is the mean $\pm S$.E. of nerve cross-sectional area determined from scanning electron microscopy of normal nerves. Counts of ectopic filiform spines were made six to eight months after surgery designed to innervate the rat tongue with the mylohyoid nerve ( $\mathrm{MH}, n=5$ rats), the lingual nerve $(\mathrm{L}, n=5)$, the lingual and mylohyoid nerves $(\mathrm{L}+\mathrm{MH}, n=5)$, or the lingual and auriculotemporal nerves $(\mathrm{L}+\mathrm{AT}, n=4)$. The filled triangle represents the incidence of ectopic filiform spines six months after removal of the chorda-lingual nerve ( $-\mathrm{CL}, n=6$ rats). From scanning electron microscopy measurements the chorda tympani has a cross-sectional area of $2100 \pm 5 \mu \mathrm{m}^{2}, n=3$. Areas are uncorrected for shrinkage and the small percentage variation in the amount of connective tissue. The correlation coefficient for the least-squares best-fit straight line is +0.991 with a $y$-intercept of $57.9 \%$ and an $x$-intercept of $125,800 \mu \mathrm{m}^{2}$.

remnant. The ectopic spines were determined to be filiform spines on the basis of their shape, structure. and pattern of keratin-like immunoreactivity. It is unlikely that filiform papillae were mistakenly classified as spine-bearing fungiform papillae, given the atypical shape or orientation of many ectopic spines, and more importantly given the conservative stipulation that, to be tallied, a spine-bearing fungiform papilla must have had at least twice the transverse area as the largest neighboring filiform papilla. Indeed, it is likely the stringency of this size criterion resulted in the converse error of failing to tally some of the smallest fungiform papillae with an ectopic spine. This could explain why, six to eight months after surgery, a mean of only 70 fungiform papillae were counted ( $n=7$ rats) compared with a mean of 87 fungiform papillae present on the normal side of the tongue $(n=19)$. If this were true, that ectopic filiform-like spines prevented detection of some small fungiform papillae. then the number of untallied fungiform papillae might be expected to increase in those instances when ectopic spines were abundant. In particular, there were $18 \pm 10$ untallied or missing fungiform papillae and a $61 \%$ incidence of ectopic spines six months after interruption of the chordalingual nerve, compared with $9 \pm 3$ missing fungiform papillae and a $38 \%$ spine incidence six to eight months after trigeminal innervation (mean \pm S.D.: $P<0.01, t$-test of means of missing fungiform papil- 
lae for $-\mathrm{CL}$ vs all Vth nerve groups at six to eight months). Thus, while all lines of evidence consistently indicated that the majority of fungiform papillae lacking a taste bud developed an ectopic filiform spine, our conservative counting procedure for tallying spine-bearing fungiform papillae probably underestimated the actual number of fungiform papillae with an ectopic spine.

The much greater effectiveness of chorda tympani axons than trigeminal axons in preventing the expression of ectopic filiform spines, suggests that it was the gustatory axons of the chorda tympani nerve that suppressed ectopic spines, either by direct action or indirectly via taste buds. If a few gustatory axons could directly suppress ectopic spines, it might explain the absence of prominent spines from $39 \%$ of empty fungiform papillae six months after interruption of the chorda-lingual nerve. Trigeminal axons were probably not numerous enough to account for this lack of spine outgrowth. Given the slope of the line in Fig. 5, trigeminal innervation equivalent to about five mylohyoid nerves or two lingual nerves would be required to lower the incidence of ectopic filiform spines from 100 to $61 \%$. Yet innervation by even one mylohyoid nerve supplied many more axons than were present in the $39 \%$ of empty fungiform papillae that lacked a spine.

It might be argued the real stimulus that prompted ectopic spines was not the loss of taste axons, but the mechanical misuse or disuse of the tongue caused by partial denervation. Perhaps improper oral motor performance promoted the extension of ectopic spines through decreased abrasion or through increased friction on the tongue surface (analogous to callus formation). If so, it is difficult to explain how abnormal motor performance would have so selectively controlled which fungiform papillae developed ectopic spines. By one month after surgery, spines were absent from all contralateral fungiform papillae as well as from scattered ipsilateral fungiform papillac with a taste bud. Further, with only one chorda tympani removed and all other nerves intact, sensory guidance of tongue movements should have been virtually normal, yet roughly half of the empty fungiform papillae in this experimental subgroup (L) had an ectopic spine. Finally, spine-like outgrowths have been reported to emerge from putative fungiform papillae of explanted, and therefore immobile, fragrnents of fetal tongue. ${ }^{4}$

In contrast to embryonic fungiform papillae that seemed to be prompted by denervation to express spines, embryonic filiform papillae continued their normal development in vitro and formed ordinary filiform spines while denervated.' Thus, the results from the organ culture of embryonic tongue fragments lend support to the inference of the present study that, after denervation, filiform spines are the default condition for both filiform and fungiform papillae. Hence, the observation of a taste bud associated with a fliform spine within cultured tongue fragments is now explicable as a taste bud-bearing fungiform papilla that subsequently developed an ectopic filiform spine in culture. ${ }^{18}$

\section{CONCLUSION}

The effects of denervation and reinnervation indicate that taste axons, and to a modest extent lingual nerve axons, negatively regulate ectopic filiform spines by suppressing spine outgrowth from the apical surface of a normal fungiform papila. This evidence and studies of cultured tongue fragments suggest an expansion of the taste axon's trophic role to include a lifelong capacity to suppress ectopic filiform spines on fungiform papillae in addition to promoting the original development ${ }^{7}$ and adult maintenance ${ }^{14}$ of fungiform taste buds. More generally, at sites of superficial cutaneous receptor cells, such as fungiform taste buds and several types of mechanoreceptors, it appears that neurotrophic dependence and the negative regulation of keratinization provide a selective evolutionary advantage by enabling denervated tissue to eliminate secondary receptor cells and adopt a more highly protective keratinized state. ${ }^{15}$ For example, after denervation of hairy skin of rats, the majority of Merkel cells degenerate and their cutaneous touch domes become much more heavily keratinized and thus better protected. ${ }^{12}$ Similarly, after the denervation-induced loss of taste buds has eliminated the main sensory function of a fungiform papilla, negative neural regulation of ectopic filiform spine formation will lead to the outgrowth of an ectopic spine that protectively covers the fungiform papilla. Additional studies will be required to determine whether taste axons prevent ectopic spines by locally neutralizing a spine promoting tissue factor, analogous to the proposal that innervation density is stabilized when cutaneous axons branch sufficiently to neutralize a tissue factor that promoted the axonal sprouting. ${ }^{2}$

Acknowledgements--This work was supported by NIH Javits Award DC00083.

\section{REFERENCES}

1. Baratz R. S. and Farbman A. I. (1975) Morphogenesis of rat lingual filiform papillae. Am. J. Anat. 143, 283-302,

2. Diamond $J$, Cooper E., Turner C. and Macintyre L. (1976) Trophic regulation of nerve sprouting. Science 193, $371-377$.

3. Farbman A. 1. (1970) The dual pattern of keratinization in fliform papillae on rat tongue. J. Anat. 106, 627-646.

4. Farbman A. 1, (1971) Differentiation of foetal rat tongue homografts in the anterior chamber of the eye. Archs oral Bioi. 16, $51-57$. 
5. Farbman A. 1. (1973) Differentiation of lingual filiform papillae of the rat in organ culture. Archs oral Biol. 18, $197-202$.

6. Gomez-Ramos P.. Leon-Feliu E. and Rodriguez-Echandia E. L. (1979) Taste buds in vallate papillae grafted to the anterior chamber of the eye. Anat. Embryol 156, 217-224.

7. Hosley M. A., Hughes S. H. and Oakley B. (1987) Neural induction of taste buds. $J$. comp. Netrol. 260, $224-232$.

8. Hume W. S. (1983) Stem cells in oral epitheha. In Stem Cells: their ldentification and Characterisation (ed. Potten C. S.), pp. 232-270. Churchill Livingston, New York.

9. Hume W.S. and Potten C. S. (1976) The ordered columnar structure of mouse fliform papillae. J. Cell Sei. 22, 149-160.

10. Kutuzov H. and Sicher H. (1951) The filiform and conical papillae of the tongue in the white rat. Anat. Rer. 110, $275-288$.

11. Mbiene J. P. and Farbman A. I. (1990) Early development of fungiform papillae in rat embryos. Chem. Senses (abstract) (in press).

12. Nurse C. A., Maclntyre L. and Diamond J. (1984) A quantitative study of the time course of the reduction in merkel cell number with denervated rat touch domes. Neuroscience 11, $521-533$.

13. Oakley B. (1970) Reformation of taste buds by crossed sensory nerves in the rat's tongue. Acta physiol. scand. 79, 88-94.

14. Oakley B. (1985) Trophic competence in mammalian gustation. In Olfactory and Gustatory Infuences on the Ceniral Nervots System (ed. Pfaff D.), pp. 92-103. The Rockefeller University Press, New York.

15. Oakley B. (1990) On the neurotrophic support of sensory receptor cells. In Olfaction and Taste X (ed. Doving K. B.) (in press).

16. Potten C. S. and Allen T. D. (1975) Control of epidermal proliferative units (EPUs). An hypothesis based on the arrangement of neighboring differentiated cells. Differentiation 3, 161-165.

17. Winkelmann R. K. (1960) Nerve Endings in Normal and Pathologic Skin. Thomas, Springfield.

18. Zalewski A. A. (1972) Regeneration of taste buds after transplantation of tongue and ganglia grafts to the anterior chamber of the eye. Expl Newrol. 35, 519-528. 